\title{
Finite amplitude standing waves in metallic rods ${ }^{\text {a) }}$
}

\author{
C. Campos-Pozuelo and J. A. Gallego-Juárez \\ Instituto de Acústica, Serrano, 144, 28006 Madrid, Spain
}

(Received 4 March 1994; accepted for publication 5 September 1994)

\begin{abstract}
Large-amplitude extensional standing waves in metals are studied theoretically and experimentally. Starting from the nonlinear elastodynamic equation for perfectly elastic solids, a one-dimensional second-order model is formulated for resonant rods. Losses are neglected and superposition of forward and backward waves is assumed. Spatial distributions of the force and particle velocity are obtained theoretically as well as the waveforms. The experimental work consists of measuring and analyzing the vibration signal along the length of cylindrical rod samples of a titanium alloy at constant temperature. Stepped rod samples are used to achieve higher strain amplitudes. The samples are driven by means of a piezoelectric transducer. The vibration amplitudes and waveforms are monitored by using a nonintrusive laser vibrometer. From a comparison between the experimental and theoretical results a value for the nonlinearity parameter of the titanium alloy is derived.
\end{abstract}

PACS numbers: 43.25.Dc, 43.25.Gf

\section{INTRODUCTION}

In this paper we present a theoretical and experimental study of finite-amplitude extensional standing waves in resonant rods of perfectly elastic isotropic solids.

It is well known that the vibration of elastic solids can be described by linear laws only in the case of infinitesimal amplitudes. In fact, if the vibration is of finite amplitude, the strain and stress tensors contain higher-order terms and they are no longer linearly related. ${ }^{1,2}$ As a consequence, the equations of motion become nonlinear ${ }^{3,4}$ and the solid medium must be characterized by higher-order elastic constants. Materials used in high-power ultrasonic transducers, such as titanium alloys, which are subjected to very large mechanical displacements, have to be studied in the nonlinear range.

The classical acoustic methods to study the dynamic elastic properties of solids under linear conditions involve the measurement of velocity and attenuation. The applied measurement procedure depends on the frequency range. For frequencies up to about $100 \mathrm{kHz}$ the usual method is to excite a resonant mode in a specimen and to measure the frequency and the resonant curves. For higher frequencies, pulse methods are better suited because the dimensions required for the specimen to be resonant would be too small.

Methods for studying the nonlinear dynamic properties of solids are not so well established. In fact, for many years nonlinear studies were mainly focused on fluid media. ${ }^{5}$ More recently interest in the study of finite-amplitude propagation in solids has grown as a consequence of various practical problems such as the propagation of seismic waves in rocks or the increasing power used in sonic and ultrasonic applications where the nonlinear properties of the solids cannot been ignored. ${ }^{6,7}$ In the literature there exist a number of theoretical and experimental investigations which determine the nonlinearity parameters of solids. ${ }^{4,6-10}$ Two main acoustic methods for studying the nonlinear elastic properties of solids have

a) Part of this research was presented at the 13 th International Symposium on Nonlinear Acoustics, Bergen, Norway, 28 June-2 July 1993. been proposed. One of the procedures consists of applying a high static stress (hydrostatic or uniaxial) and measuring the time for small-amplitude waves to travel one round trip in the sample. ${ }^{11,12}$ The velocity slope versus static stress is related to the second- and third-order elastic coefficients. ${ }^{13,14} \mathrm{~A}$ recently modified version of this method based on the changes in frequency of small-amplitude standing waves in a rod as a function of an applied high uniaxial uniform stress. ${ }^{8}$ The other method employed consists in generating a sinusoidal signal at the boundary of the material sample and in measuring the nonlinear distortion of the wave along the propagation path. The nonlinear elastic properties are inferred from the growth of the harmonics. Generally, the studies were restricted to the second harmonic and they were based on one-dimensional models under the assumption of semi-infinite media. ${ }^{3,4,15}$ The applied equations are similar to those developed for finite amplitude waves in fluids. ${ }^{3,10,16-18}$ This procedure requires the use of high ultrasonic frequencies to generate progressive waves under bulk propagation conditions. Few analyses on finite amplitude standing waves are found in the literature. Some papers describe the behavior of nonlinear standing acoustic waves in fluids. ${ }^{19,20}$ However, nonlinear vibrating elastic solids in resonance have only recently found attention in some investigation. Johnson et al. ${ }^{7}$ have presented a work where the nonlinearity parameters of rocks were measured by studying the resonant response of a bar subjected to finite amplitude deformations. The method was based on the measurement of resonance frequency variations with changing vibration amplitude; no information was given about the harmonic distribution along the geometry of the sample. This method, which was applied to highly nonlinear materials (rocks) by using very low frequencies $(\approx 1$ $\mathrm{kHz}$ ), was not sensitive enough for studying nonlinearities in metals.

This article deals with a theoretical and experimental study of the nonlinear behavior of metals subjected to highintensity ultrasonic stresses. The experimental study was carried out with resonant specimens employing longitudinal vi- 
brations at frequencies of about $23 \mathrm{kHz}$. The material employed in this study was a titanium alloy (Ti 6 Al 4 V) commonly used in the construction of high-power ultrasonic transducers. ${ }^{21}$ The theoretical model developed was based on an one-dimensional approach of the nonlinear elasticity theory, neglecting losses and assuming superposition of forward and backward waves.

\section{THEORY}

This study considers longitudinal waves in an isotropic rod with a small $d / \lambda$ ratio ( $d$ being the cross-sectional diameter and $\lambda$ the wavelength). Under these conditions it is assumed that the vibration of the specimen can be described by a one-dimensional model. In the present approach losses are neglected. This assumption can be justified by taking into account that the solids studied are polycrystalline metals in which the dissipation energy is small. The excitation amplitude stays within the range where no structural changes in the material are produced to maintain elasticity conditions. Only second-order terms of the strain are considered.

\section{A. Second-order one-dimensional wave equation}

Finite-amplitude vibrations involve sufficiently large deformations that the ordinary (linear) theory of elasticity is not valid. Theories for finite deformations differ in two main points from the infinitesimal theory. First, the spatial or Eulerian coordinates are not interchangeable with the material or Lagrangian coordinates. Second, the expression of the strain energy contains higher-order terms and, therefore, more coefficients have to be defined to describe the solid. ${ }^{1,2,4,10}$ In this paper all equations are expressed in Lagrangian coordinates. We call $x$ the coordinate along the axis of the rod. The system is considered to be at constant temperature and, following from this, all the elastic constants involved stay at constant temperature. As mentioned above, we consider an isotropic material in which only longitudinal plane waves are propagating; therefore only the stress and strain components along the axis of the rod appear in the partial differential equation of the wave motion. Note that this assumption does not mean zero transverse strain as in a semi-infinite solid. The free energy $x$ of the system related to this strain component can be written as ${ }^{10,22}$

$$
\rho_{0} \chi=\frac{1}{2} C_{1} \eta^{2}+\frac{1}{6} C_{2} \eta^{3},
$$

where $\rho_{0}$ is the density in the initial state, $C_{1}$ and $C_{2}$ are the one-dimensional compressional, second- and third-order elastic constants, and $\eta$ the strain component along the axis of the rod which can be expressed in Lagrangian coordinates as

$$
\eta=\frac{\partial u}{\partial x}+\frac{1}{2}\left(\frac{\partial u}{\partial x}\right)^{2}
$$

with $u$ being the displacement along the axis of the rod. Taking into account that only axial stress is applied to the rod, one may express the elastic constants in terms of the Lamé coefficients $(\lambda$ and $\mu$ ) and Murnaghan coefficients ( $l$, $m$, and $n$ ) for an isotropic solid in the form: ${ }^{1}$

$$
\begin{aligned}
& C_{1}=\frac{\mu(3 \lambda+2 \mu)}{\lambda+\mu}, \\
& C_{2}=\frac{\mu^{3}}{(\lambda+\mu)^{3}} l+\frac{\mu(3 \lambda+2 \mu)^{2}}{2(\lambda+\mu)^{3}} m+\frac{3 \lambda^{2}}{4(\lambda+\mu)^{3}} n .
\end{aligned}
$$

By using Eq. (2) the Piola-Kirchhoff tensor $(T)^{22}$ can be written in the form

$$
T=C_{1} \frac{\partial u}{\partial x}+\frac{3 C_{1}+C_{2}}{2}\left(\frac{\partial u}{\partial x}\right)^{2} .
$$

Conservation of linear momentum leads to the following nonlinear wave equation: ${ }^{4}$

$$
\rho_{0} \ddot{u}=\frac{\partial T}{\partial x}=Y_{0} \frac{\partial^{2} u}{\partial x^{2}}+Y_{1} \frac{\partial}{\partial x}\left[\left(\frac{\partial u}{\partial x}\right)^{2}\right]
$$

where $Y_{0}=C_{1}$ and $Y_{1}=\left(3 C_{1}+C_{2}\right) / 2$. The nonlinear behavior of the material is usually described by the nonlinearity parameter $\beta$ which can be defined as the ratio of the coefficient of the nonlinear term to the linear term in the nonlinear wave equation, that is,

$$
\beta=Y_{1} / Y_{0}
$$

Several approximate procedures are known to obtain solutions of Eq. (4). ${ }^{23}$ Here we use the method of successive approximations in which the solution is assumed as an addition of two terms in the form: $u=u_{l}+u_{n l}$, where $u_{l}$ represents the first-order approximation and $u_{n l}$ the second-order nonlinear approximation. Substituting $u$ in Eq. (4) we obtain the equation

$$
\rho_{0} \ddot{u}_{l}-Y_{0} \frac{\partial^{2} u_{l}}{\partial x^{2}}=0
$$

for the first-order approximation, and the equation

$$
\rho_{0} \ddot{u}_{n l}-Y_{0} \frac{\partial^{2} u_{n t}}{\partial x^{2}}=Y_{1} \frac{\partial}{\partial x}\left[\left(\frac{\partial u_{l}}{\partial x}\right)^{2}\right]
$$

for the second-order approximation.

For a plane longitudinal wave traveling in the forward $x$ direction, the solution of the first equation has the form $u_{l}=A e^{j(\omega t-k x)}$, with $A$ being the amplitude of the displacement at the origin, $\omega$ the angular frequency, and $k$ the wave number. The solution of Eq. (6) results to be

$$
u_{n l}=\left(Y_{1} k^{2} / 2 Y_{0}\right) A^{2} x e^{j 2(\omega t-k x)} .
$$

Consequently, the solution of Eq. (4) in the second-order approximation for a longitudinal harmonic wave traveling in the forward direction is of the form

$$
u_{a}=A e^{j(\omega t-k x)}+p A^{2} x e^{j 2(\omega t-k x)},
$$

where $p=Y_{1} k^{2} / 2 Y_{0}$.

\section{B. Nonlinear extensional waves in resonant systems}

The main objective of this work is to study nonlinear extensional vibrations in resonant systems such as finite rods. To approach this problem we consider a longitudinal plane wave traveling along the axis of the rod, incident on the boundary where it is reflected in opposite direction. There- 
fore, to set up a standing wave pattern we assume superposition of two waves of finite amplitude traveling in forward and backward direction, i.e.,

$$
u=u_{a}+u_{b} \text {. }
$$

The expression for the forward wave $u_{a}$ is shown in Eq. (8) while the expression for the backward wave takes the form

$$
u_{b}=B e^{j(\omega t+k x)}+p B^{2}(l-x) e^{j 2(\omega t+k x)},
$$

where $l$ is the length of the $\operatorname{rod}$ and $A$ and $B$ are constants to be determined by the boundary conditions. If the system is excited by a sinusoidal source, the boundary conditions require that

$$
\begin{aligned}
& u(x=0, t)=u_{0}(t)=u_{1} e^{j \omega t}, \\
& T(x=0, t)=T_{0}(t)=T_{1} e^{j \omega t},
\end{aligned}
$$

where $u_{1}$ and $T_{1}$ are the amplitudes of the displacement and the stress at the input point. Applying these boundary conditions and neglecting again all third- and higher-order terms of $\partial u / \partial x$, we obtain for the displacement and the stress

$$
\begin{aligned}
u(x, t)= & u_{0}(t) \cos k x+\frac{T_{0}(t)}{k Y_{0}} \sin k x-p \frac{l u_{0}(t)^{2}}{4} g(x) \\
& +p \frac{T_{0}(t)^{2}}{4 k^{3} Y_{0}^{2}} h(x)+p \frac{T_{0}(t) u_{0}(t)}{2 k^{2} Y_{0}} m(x), \\
\frac{T(x, t)}{Y_{0}}= & \frac{T_{0}(t)}{Y_{0}} \cos k x-k u_{0}(t) \sin k x \\
& -p \frac{k l u_{0}(t)^{2}}{4} g_{F}(x)+p \frac{T_{0}(t)^{2}}{4 k^{2} Y_{0}^{2}} h_{F}(x) \\
& +p \frac{T_{0}(t) u_{0}(t)}{2 k Y_{0}} m_{F}(x),
\end{aligned}
$$

where

$$
\begin{aligned}
g(x)= & \cos k x+2 j \sin k x-\cos 2 k x-j \sin 2 k x \\
& +j \frac{2 x}{l} \sin 2 k x,
\end{aligned}
$$

$h(x)=k l \cos k x-8 \sin k x+j 2 k l \sin k x-k l \cos 2 k x$

$-j k l \sin 2 k x+2 k x \sin 2 k x$,

$$
m(x)=j k l \cos 2 k x-2 k l \sin k x-j 2 \sin k x
$$

$+j 2 k x \cos 2 k x-j k l \cos 2 k x+k l \sin 2 k x$,

$g_{F}(x)=2 j \cos k x-\sin k x+2 \sin 2 k x-j 2 \cos 2 k x$

$$
+j \frac{4 x}{l} \cos 2 k x+\frac{2}{k l} \sin 2 k x+\frac{8 \sin ^{2} k x}{k l},
$$

$h_{F}(x)=-k l \sin k x-8 \cos k x+j 2 k l \cos k x$

$-2 k l \sin 2 k x-j 2 k l \cos 2 k x+2 \sin 2 k x$

$+4 k x \cos 2 k x+8 \cos ^{2} k x$,

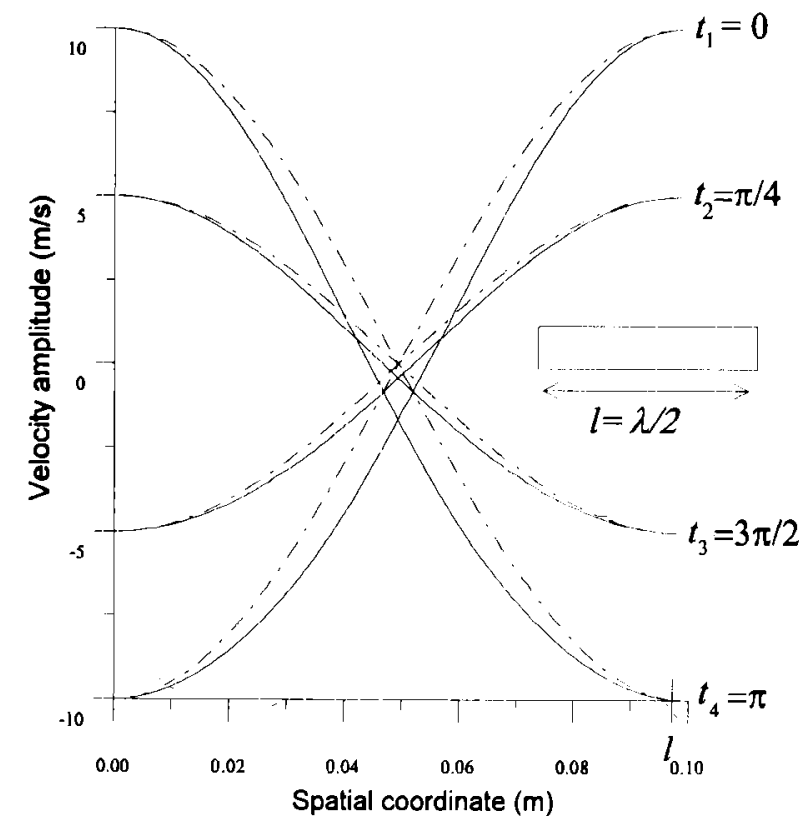

FIG. 1. Theoretical distribution of the linear (_- ) and nonlinear (particle velocity at different times within a period for a cylindrical rod $(l=\lambda / 2)$.

$$
\begin{aligned}
m_{F}(i x)= & -2 j k l \sin 2 k x-2 k l \cos k x-j 2 \cos k x \\
& +j 2 \cos 2 k x-4 k x \sin 2 k x+j 2 k l \sin k x \\
& +2 k l \cos 2 k x-4 \sin 2 k x .
\end{aligned}
$$

For comparison with experimental data it is more useful to express Eqs. (12) and (13) in terms of particle velocity and force, i.e.,

$$
\begin{aligned}
\dot{u}(x, t)= & \dot{u}_{0}(t) \cos k x+j \frac{F_{0}(t)}{Z} \sin k x \\
& +j n \frac{k l \dot{u}_{0}(t)^{2}}{2} g(x)+j n \frac{F_{0}(t)^{2}}{2 Z^{2}} h(x) \\
& +n \frac{F_{0}(t) \dot{u}_{0}(t)}{Z} m(x), \\
F(x, t)= & F_{0}(t) \cos k x+j \dot{u}_{0}(t) Z \sin k x \\
& +n k l Z \frac{\dot{u}_{0}(t)^{2}}{4} g_{F}(x)+n \frac{F_{0}(t)^{2}}{4 Z} h_{F}(x) \\
& -j n \frac{F_{0}(t) \dot{u}_{0}(t)}{2} m_{F}(x),
\end{aligned}
$$

where $n$ is defined as $n=p / k \omega$ and $Z$ is the impedance of the rod, $Z=\rho_{0} c S$, with $c$ being the sound propagation velocity $\left(c^{2}=Y_{0} / \rho_{0}\right)$.

We can apply these results to the simple case of a free cylindrical rod of uniform cross section with length $l$ corresponding to the linear resonance condition $l=(\lambda / 2)$, with $\lambda$ being the wavelength. The results obtained for the particle velocity and the force are shown in Figs. 1 and 2 for a rod made of Ti $6 \mathrm{Al} 4 \mathrm{~V}\left(c=4750 \mathrm{~m} / \mathrm{s}, \rho_{0}=4400 \mathrm{~kg} / \mathrm{m}^{3}\right)$. The value of the nonlinearity parameter has been arbitrary taken as $\beta=50$. Note that the maximum harmonic distortion occurs 


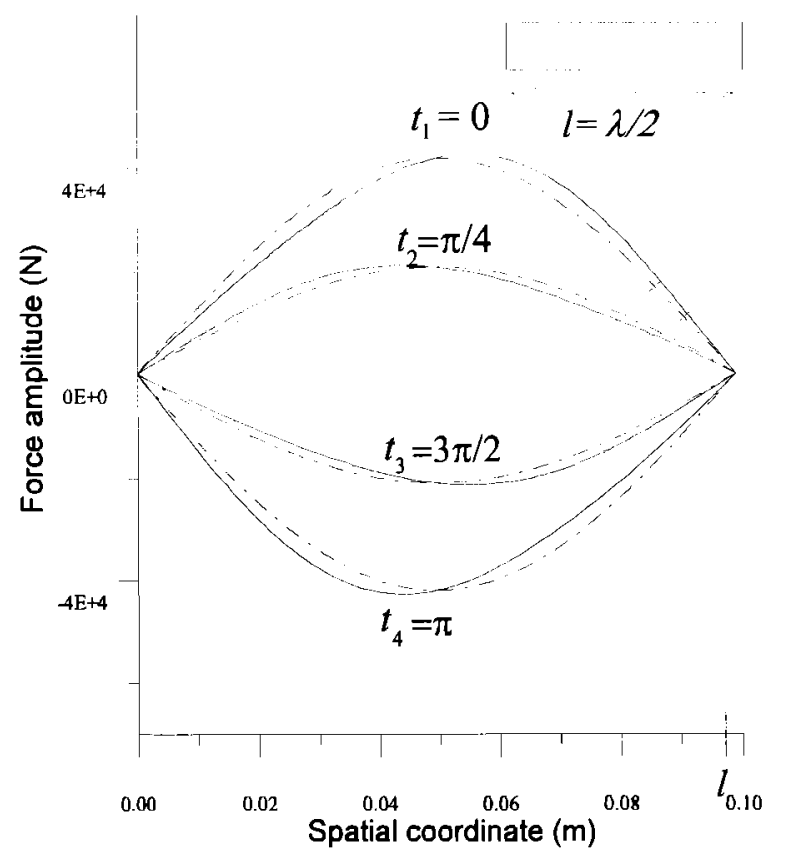

FIG. 2. Theoretical distribution of the linear (-_) and nonlinear (force distribution at differents times within a period for a cylindrical rod $(l=\lambda / 2)$.

in the center of the rod while at the end of the rod no harmonic generation is found. This behavior is similar to that obtained by Dah-You ${ }^{20}$ for the nonlinear resonance of a onedimensional air-filled tube. We find that in the linear behavior the node is perfectly defined, while in the second-order approximation the point of zero vibration amplitude changes with time, i.e., it is not a real node. This result follows from the fact that amplitude of the second harmonic is maximum at the node of the fundamental.

Taking into account that in any case it is possible to write $F_{1}=Z_{i} \dot{u}_{1}$, with $Z_{i}$ being the mechanical impedance at $x=0$, Eq. (14) can be written in the form

$$
\dot{u}(x, t)=\dot{u}_{1} e^{j \omega t} a(x)+\dot{u}_{1}^{2} e^{j 2 \omega t} \beta b(x) .
$$

Equation (16) shows that for every $x$ value the amplitude of the second harmonic, $\dot{u}_{1}^{2} \beta b(x)$, follows a parabolic behavior with respect to the amplitude of the excitation velocity $\left(\dot{u}_{1}\right)$. The coefficient of this parabola is proportional to the nonlinearity parameter $\beta$ of the material and dependent on the spatial coordinate in the rod. Computing this parabola for different points of the rod and fitting the computed curves with the experimentally obtained data, the value of the nonlinearity parameter $\beta$ for the material can be determined. In this way a new procedure for calculating the nonlinearity parameter $\beta$ is proposed. The practical application of this method is presented in Sec. III.

\section{EXPERIMENTS}

The experimental setup for measuring amplitude and frequency components of the particle velocity at different points along resonant specimens is shown in Fig. 3. It consists of a driving system to excite the samples at resonance and data acquisition equipment.

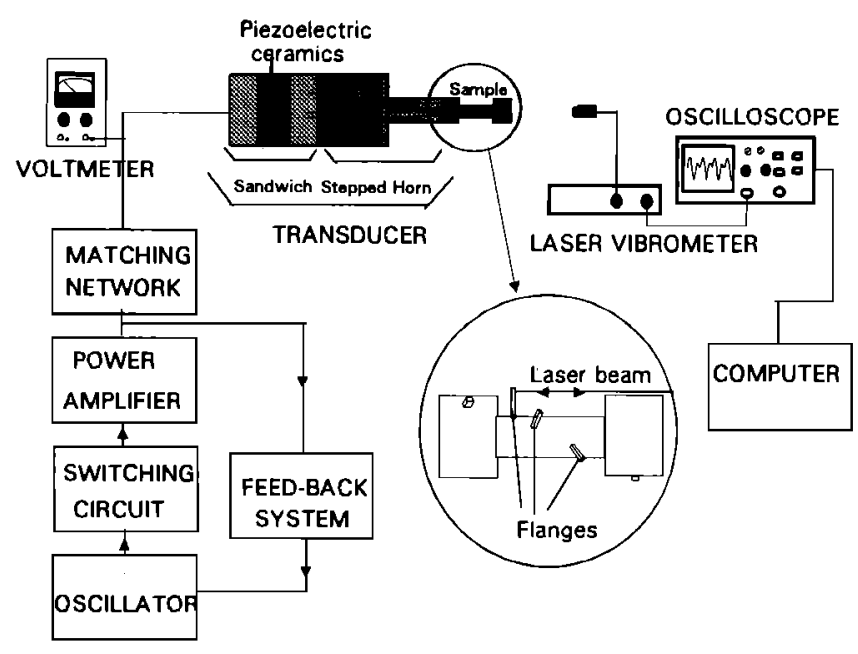

FIG. 3. Experimental setup.

\section{A. Excitation system}

The excitation system consisted of a specially designed electronic generator and a piezoelectric transducer. The electronic generator used to drive the transducer implemented a feedback system to automatically adjust the excitation frequency to the transducer's resonant frequency. ${ }^{24}$ In addition, a switching circuit was designed to produce periodic interruptions in the excitation signal; in such a way the time of excitation could be controlled to keep the temperature of the sample constant. This circuit essentially consisted of a programmable counter and an output stage which acts on the power amplifier input signal. ${ }^{25}$ The excitation driving time could be varied between 1.5 and $9.6 \mathrm{~s}$ and the off time between 1.5 and $180 \mathrm{~s}$. Within these ranges it was possible to keep the temperature constant for all the samples and excitation levels studied. In addition, a resistive network which matched the generator to the load was inserted at the output of the electronic generator to increase the stability of the system by widening the electric bandwidth.

The driving transducer, a resonant system at about 23 $\mathrm{kHz}$, was constructed from an assembly of two half-wave elements: A piezoelectric sandwich and a stepped horn (Fig. 3). The sandwich element consisted of four piezoelectric ceramics between two metallic cylindrical rods. A damping material (silicone rubber) was attached at the backing rod to increase the mechanical bandwidth of the system and to improve the system stability. The stepped horn acted as a mechanical amplifier to achieve higher vibration amplitude at its thinner termination where the sample was attached.

\section{B. Samples}

Cylindrical stepped titanium alloy ( $\mathrm{Ti} 6 \mathrm{Al} 4 \mathrm{~V}$ ) samples with thinned-out central sections were designed to achieve higher strain within the material (Fig. 4). The samples were sized to meet the resonance condition of the transducer. In deriving the equations for this sample shape, the resonance condition $\tan (\omega L / 4 c)=d_{2} / d_{1}$ is obtained, ${ }^{26}$ where $\omega$ is the resonance frequency, $L$ the length of the sample, $c$ the sound velocity, and $d_{2}$ and $d_{1}$ the diameters of the central and the end sections, respectively. A number of small rectangular 


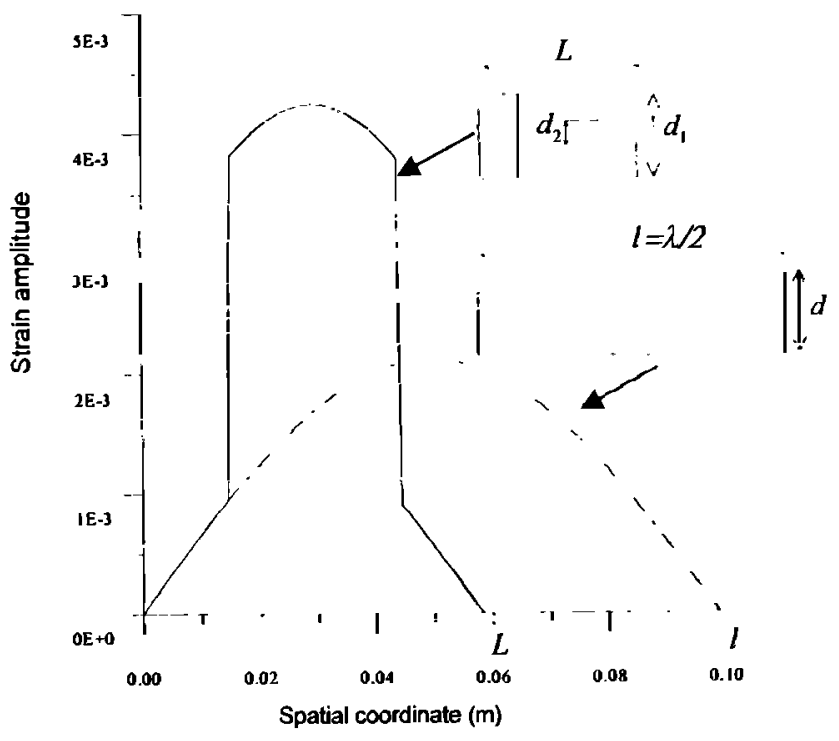

FIG. 4. Strain amplitude for a cylindrical rod with $l=\lambda / 2(\longrightarrow)$, and stepped rod with a ratio $d_{1} / d_{2}=2(\longrightarrow)$.

flanges along the length of the samples (Fig. 3) allowed the measurement of the longitudinal vibration at different points of the sample. Because of their small size with respect to the wavelength the flanges did not affect the impedance or the resonance frequency of the sample. The flanges were sized so that their flexural modes were not close to the frequency of excitation. For comparison, uniform cylindrical samples were used to determine the linear range of the transducer (Fig. 4). All the measurements were carried out by keeping the excitation of the driving transducer within this linear range. However, the stepped samples vibrated nonlinearly under these conditions due to the high strain amplitude in their central section. ${ }^{26,27}$

\section{Data acquisition system}

The amplitude of the displacement was measured by using a vibrometer based on a He- $\mathrm{Ne}$ laser interferometer. The vibrometer measures frequencies of up to $1.5 \mathrm{MHz}$ and particle velocities between $10 \mu \mathrm{m} / \mathrm{s}$ and $10 \mathrm{~m} / \mathrm{s}$. The vibrometer was connected to a PC 386 computer to store and analyze the waveshapes. In the analysis FFT methods were applied. The temperature of the sample was monitored by an infrared thermometer to avoid load through a contact point. ${ }^{28}$

\section{RESULTS}

This section presents the experimental results, their comparison with theoretical predictions, and the determination of the nonlinearity parameter of the studied material.

To verify the assumption of one-dimensional behavior, measurements of the linear strain along the stepped rod were conducted and the obtained values were compared with the corresponding results from the theoretical one-dimensional approach (Fig. 5). The experimental strain was obtained from measurements of the transverse displacement along the length of the sample assuming that this strain is constant in

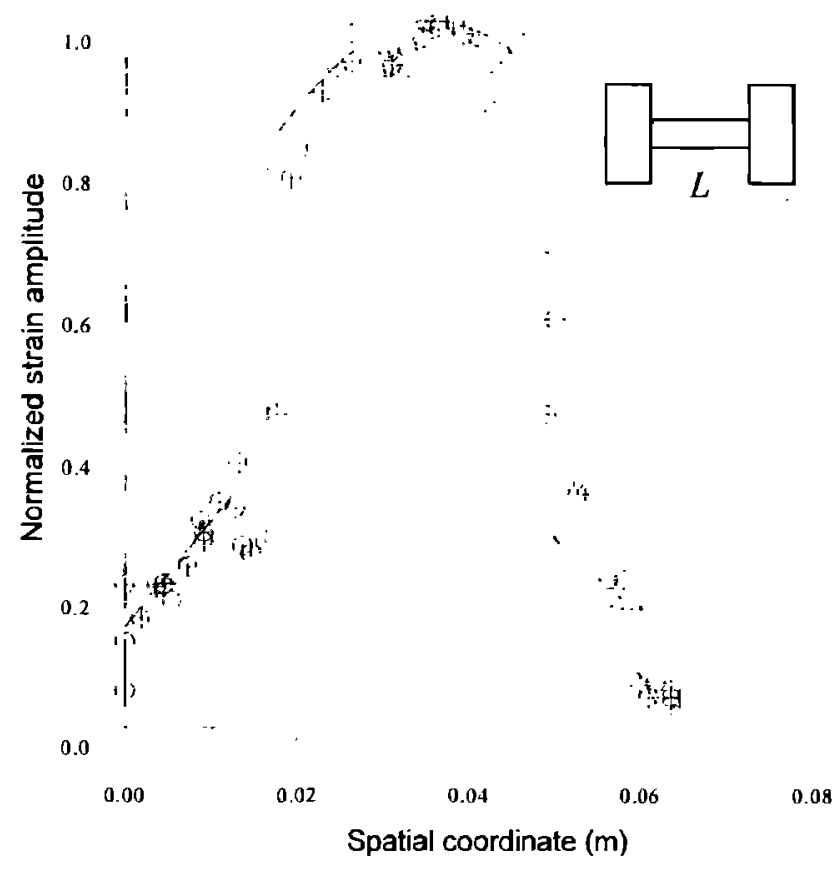

FIG. 5. Lincar strain distribution for a stepped rod with a ratio $d_{1} / d_{2}=1.6$. Theoretical (—) and experimental $(\oplus)$.

the whole cross section $(d \ll \lambda)$. Figure 5 shows good agreement between measurement and theory and confirms the validity of the assumption.

To compute the nonlinear theoretical values for the stepped rod samples described above. the boundary condjtions are defined as

$$
\dot{u}(x=0)=\dot{u}_{0}(t), \quad F(x=l)=0 .
$$

with the conditions of conlinuity of displacement and force in every change of section of the rod. Applying these conditions to Eqs. (14) and (15), the distribution of velocity and

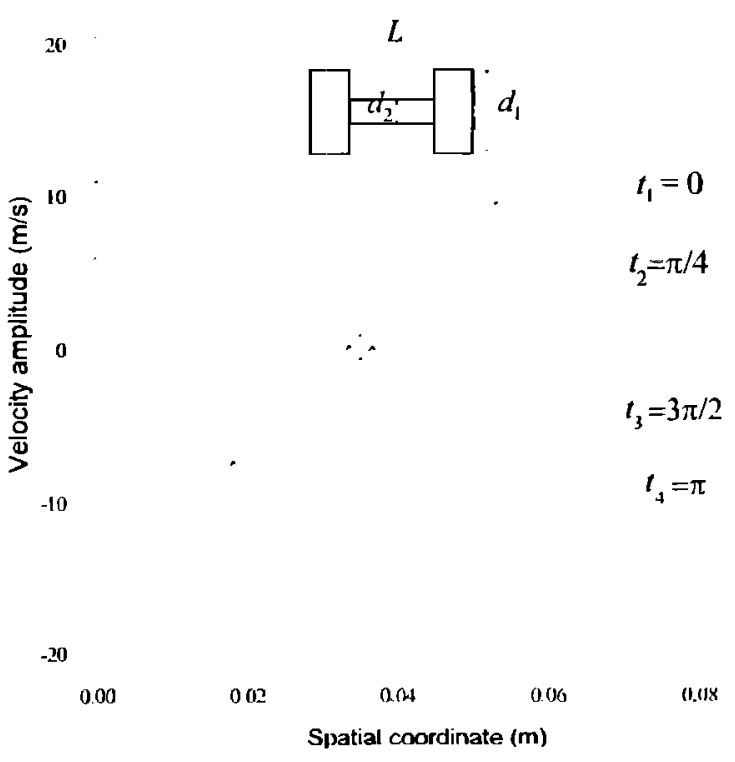

FIG. 6. Theoretical distribution of the linear (- - ) and nonlinear (- - ) particle velocity at different times within a period for a stepped rod $\left(d_{1} / d_{2}=1.6\right)$. 


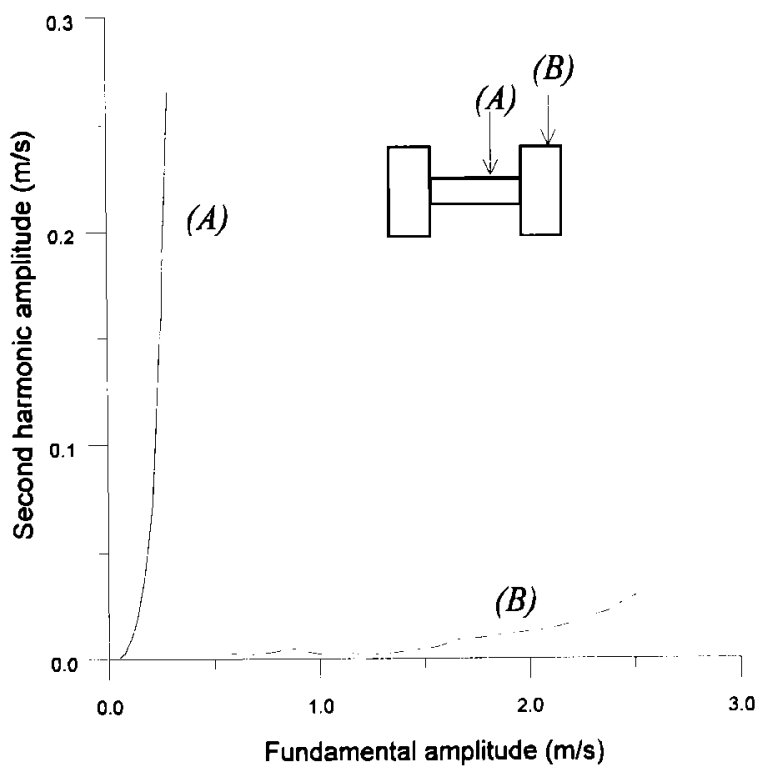

FIG. 7. Measured second harmonic versus fundamental at the thinner $(\longrightarrow)$ and thicker $(\longrightarrow)$ sections. Stepped rod $\left(d_{1} / d_{2}=1.6\right)$.

force is obtained. Figure 6 shows the calculated particle velocity distribution along the length of a Ti $6 \mathrm{Al} 4 \mathrm{~V}$ stepped rod with $d_{1} / d_{2}=1.6$. As for uniform cylindrical rods, the generation of harmonics is maximum at the central point which is not more a real node. Figure 7 shows the measured amplitude of the generated second harmonic versus the amplitude of the fundamental at two different sections of the sample. A strong parabolic generation of the second harmonic is observed at the central section as compared to minor nonlinearities at the end section. This result agrees with theoretical predictions. Figure 8 shows a comparison between experimental and calculated amplitudes of the second harmonic versus the fundamental at two different points in

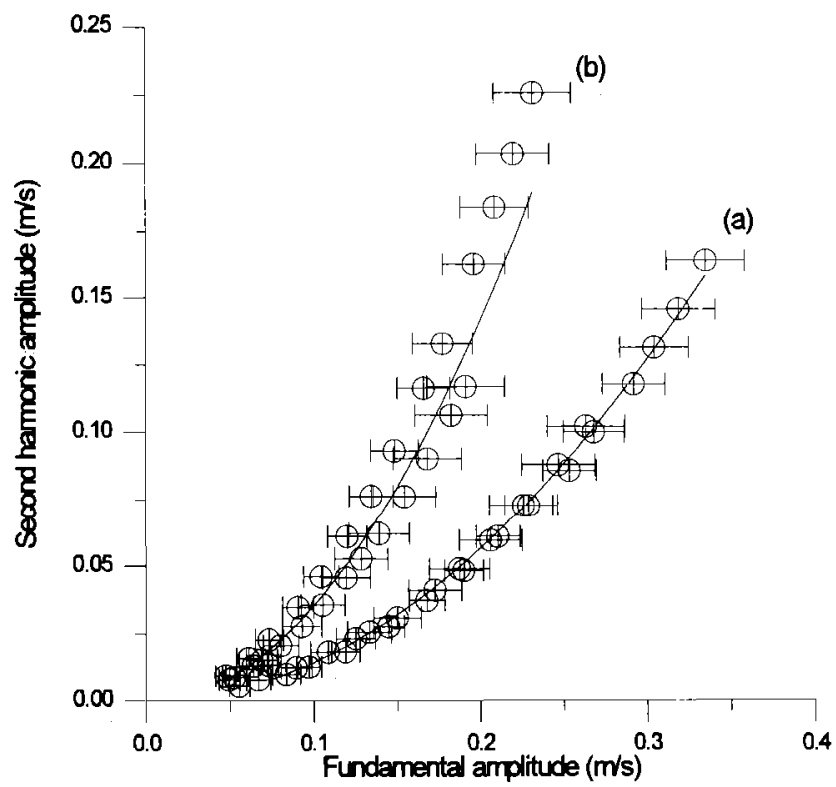

FIG. 8. Second harmonic versus fundamental at two different points in the thinner section of the stepped rod. Theoretical ( $(-)$ and experimental $(\oplus)$.

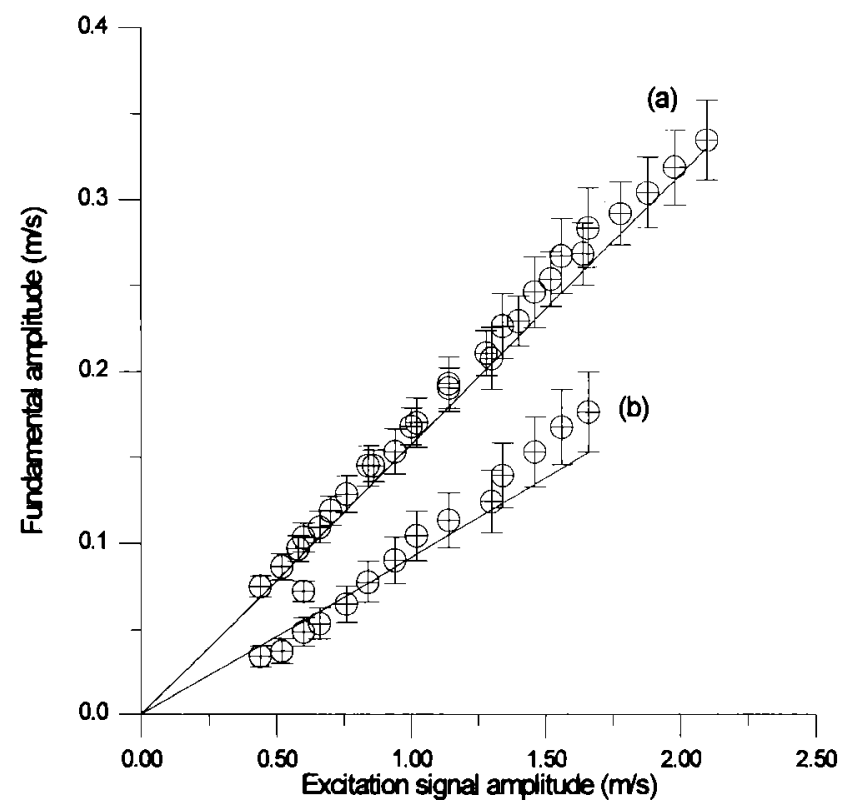

FIG. 9. Fundamental versus excitation at two different points in the thinner section of the stepped rod. Theoretical ( $\longrightarrow$ ) and experimental $(\oplus)$.

the thinned-out section of the stepped rod. All computed and measured parabolic curves were fitted by means of a single value which, according to the method proposed in this paper, corresponds to the nonlinearity parameter $\beta$ of the material. For the titanium alloy here studied this parameter resulted to be $\beta=28$.

Finally, a comparison between experimental and computed fundamental amplitudes, as a function of the excitation at the same points as in Fig. 8, is presented in Fig. 9. Again good agreement is observed.

\section{CONCLUSIONS}

Large amplitude standing waves in a titanium alloy (Ti 6 Al 4 V) were studied theoretical and experimentally. The theoretical approach consisted of a one-dimensional model based on the nonlinear elasticity theory. Superposition of two finite-amplitude waves was assumed propagating in positive and negative spatial direction. This model predicts a strong parabolic increase of the second-harmonic versus the fundamental at the central part of a resonant rod while at its end no distortion of the sinusoidal wave is expected. The experimental work was carried out with resonant, stepped samples to achieve higher strain amplitudes. The measurements showed good agreement with theoretical predictions. From curve fitting of the experimental and theoretical results a value for the compressional nonlinearity parameter $\beta$ of the material could be obtained. This method provides useful information about the nonlinear behavior of materials used for the construction of high-power ultrasonic transducers. The upper threshold of the linear range for those materials was determined as well as the vibration features of resonant structures in the nonlinear range. 


\section{ACKNOWLEDGMENTS}

This research was supported by the CICYT within the research project TAP 93-230. The authors thank Dr. F. Montoya-Vitini and P. T. Sánz-Sánchez for the design and construction of the electronic excitation system and Dr. T. Hoffmann for revision.

${ }^{1}$ F. D. Murnaghan, Finite Deformation of an Elastic Solid (Wiley, New York, 1951).

${ }^{2}$ R. N. Thurston, in Physical Acoustics, edited by W. P. Mason (Academic, New York, 1964) Vol. 1A, pp. 1-110.

${ }^{3}$ R. N. Thurston and M. J. Shaphiro, J. Acoust. Soc. Am. 41 1112-1125 (1967).

${ }^{4}$ M. A. Breazeale and J. Philip, in Physical Acoustics, edited by W. P. Mason and R. N. Thurston (Academic, Orlando, 1984), Vol. XVII, pp. 1-60.

${ }^{5}$ L. Bjornó, in Acoustics and Vibration Progress, edited by R. W. B. Stephens and H. G. Leventhal (Chapman and Hall, London, 1976), Vol. 2, pp. 101-198.

${ }^{\circ}$ P. A. Johnson, G. D. Meegan, K. R. McCall, T. J. Shankland, R. A. Guyer, and B. P. Bonner, in Advances in Nonlinear Acoustics, edited by $\mathbf{H}$. Hobaek (World Seientifie, Singapore, 1993), pp. 525-530.

${ }^{7}$ P. A. Johnson, P. Rasolofosaon, and B. Zinszner, in Ref. 6, pp. 531-536.

${ }^{8}$ A. Korotkov, M. Slavinsky, and A. Sutin, in Ref. 6, pp. 370-375.

${ }^{9}$ J. K. Na and M. A. Breazeale, in Frontiers in Nonlinear Acoustics, edited by M. F. Hamilton and P. T. Blackstock (Elsevier Science, london, 1990), pp. 571-576.

${ }^{10}$ M. A. Breazeale, in Ref. 6 (World Scientific, 1993), in Ref. 6, pp. 45I456.

${ }^{11}$ D. S. Hughes and J. L. Kelly, Phys. Rev. 92, 1145-1149 (1953).
${ }^{12}$ R. T. Smith, R. Stern, and R. W. B. Stephens, J. Acoust. Soc. Am. 40, 1002-1008 (1966).

${ }^{13}$ R. N. Thurston and K. Brugger, Phys. Rev. 133, Al604-A1610 (1964).

${ }^{14}$ R. A. Toupin and B. Bernstcin, J. Acoust. Soc. Am. 33, 216-225 (1961).

IS K. R. McCall, in Ref. 6. pp. 519-524.

${ }^{16}$ E. A. Zabolotskaya, Sov. Phys. Acoust. 32, 36-37 (1986).

${ }^{17}$ E. A. Zabolotskaya, Sov. Phys. Acoust. 32, 296-299 (1986).

${ }^{18}$ A. N. Norris and S. Kostek, in Ref. 6, pp. 463-471.

${ }^{19}$ A. L. Van Buren, J. Sound Vib. 42, 273-280 (1975).

${ }^{20}$ M. Dah-You, Chin. J. Acoust. 9, 193-204 (1990).

${ }^{21}$ J. A. Gallego-Juárez, in Power Sonic and Ultrasonic Transducers Design (Springer-Verlag. Berlin, 1988), Chap. 10, pp. 175-184.

$22 \mathrm{~J}$. J. Gagnepain, in Ultrasonic Methods in Evaluation of Inhomogeneous Materials, edited by Alippi and W. G. Mayer (Martinus Nijhoff. Dordrecht, 1987), pp. 243-262.

${ }^{23}$ M. Planat, "Propagation nonlinaire des ondes acoustiques dans les solides," These presentée a l'Université de France-Comte, Besançon, France (1984).

24 J. A. Gallego-Juárez, G. Rodriguez-Corral, J. L. San Emeterio, and F. Montoya-Vitini, European Patent NOEP 450030 (1991).

${ }^{25}$ C. Campos-Pozuelo, J. A. Gallego-Juárez, and P. T. Sanz-Sánchez, Ultrasonic International 93 Conference Proceedings (Butterworth-Heinemann, Oxford, 1993). pp. 571-574.

${ }^{2 n}$ W. P. Mason, in Low- and High-Amplitude Internal-Friction Measurements in Solids and Their Relation to Imperfection Motions, edited by C. J. Mahon, Jr. (Wiley Interscience, New York, 1968), pp. 288-382.

${ }^{27}$ C. Campos-Pozuelo and J. A. Gallego-Juárez, Ultrasonic International 91 Conference Proceedings (Butterworth-Heinemann, Oxford, 1991), pp. 645-648

${ }^{28}$ R. B. Minogna, R. E. Green, Jr., J. C. Duke. Jr., E. G. Henneke, and K. L. Reifsnider, Ultrasonics 19, 159-163 (1981). 